\title{
Influencia del bilingüismo en la estrategia cognitiva y el desarrollo cognitivo*.
}

\author{
Sandra Ben-Zeev \\ Bilingual Education Service Center
}

\section{RESUMEN}

Se plantea la hipótesis de que la interferencia mutua entre las dos lenguas de los niños bilingües les obliga a desarrollar estrategias especiales de actuación que, de alguna forma, aceleran el desarrollo cognitivo. La muestra estaba compuesta por dos grupos de niños bilingües anglo-hebreos, un grupo evaluado en los Estados Unidos y el otro en Istael, y dos grupos de niños judíos monolingües, de los cuales los evaluados en Estados Unidos sólo hablaban inglés, y los evaluados en Israel sólo hablaban hebreo. En todos los grupos la ocupación de los padres y su nivel educativo eran similares. Se calculó el $\mathrm{CI}$ a partir de cuatro subtests del WISC (semejanzas, memoria de dígitos, figuras incompletas y ordenación de imágenes) y se controló estadísticamente. A pesar del bajo nivel de vocabulario, los bilingües manifestaron un procesamiento más avanzado del material verbal, eran más discriminativos en sus distinciones perceptivas, más propensos a buscar la estructura en situaciones perceptivas, y más capaces de reorganizar sus percepciones como respuesta al feedback.

Cuando un niño crece en un ambiente bilingüe en que se superponen dos dominios del uso del lenguaje y dos conjuntos de interlocutores, se enfrenta continuamente a la interferencia entre sus dos lenguas (Weinreich, 1953). Si hay diferentes estrategias para aprender el lenguaje (Bloom, 1970), habrá que esperar que los bilingües adopten estrategias características en función de las dificultades que encuentran. En este artículo se sugiere que el niño bilingüe desarrolla

* Cbild Development, 1977, 48, pp. 1009-1018. (C) The Society for Research in Child Development, Inc. (C) de esta versión en castellano: Estudios de Psicología, 1981. 
una estrategia de lenguaje que le ayuda a resolver la interferencia interlenguas. Esta estrategia requiere un examen mayor de las entradas verbales (incluyendo la retroalimentación de su propia producción) y una mayor vigilancia de la inconsistencia en el uso de reglas o de los casos en los que el input no se aviene un sistema interpretable de reglas.

Los monolingües también necesitan mantener diferentes conjuntos de reglas de lenguaje separados entre sí, ya que una única lengua toma formas distintas cuando se aplica a diferentes registros del habla y a diferentes dominios del discurso. Pero en este caso las diferencias en las reglas son relativamente superficiales y son una cuestión de costumbre, mientras que el niño bilingüe tiene que aprender a distinguir dos sistemas diferentes de reglas de lenguaje. La estrategia más directa para evitar la interferencia entre las dos lenguas es prestar una atención especial a los aspectos sistemáticos de ambas.

Una hipótesis fundamental de este estudio es que los niños muy bilingües procesan las reglas sintácticas con una especial flexibilidad. El proceso de aprendizaje del lenguaje exige a los niños arriesgarse a hacer inferencias, algunas de las cuales son incorrectas por hipergeneralización o interpretación idiosincrática (Ervin-Tripp, 1973). Probablemente la situación bilingüe anima al niño a inferir reglas. Teniendo dos símbolos para la mayoría de los referentes, el niño bilingüe aprende pronto que las palabras son arbitrarias, y no intrínsecas. Obligado a tener medios alternativos para expresar una idea dada, se hace pronto consciente de que la relación entre una idea y su medio de expresión es problemática. El niño bilingüe tiene la experiencia de cometer muchos errores en la aplicación del lenguaje adecuado para cada situación. Probablemente también cometa más errores que los otros niños en la inferencia de reglas sintácticas, dado que su tiempo de exposición a cada lengua es menor. Como resultado de estos factores, el niño bilingüe es más consciente que los otros de que el aprendizaje de una lengua es una comprobación de hipótesis, y que requiere un esfuerzo. Está más preparado para rechazar hipótesis sintácticas cuando el feedback señala que es necesario hacerlo, y para pasar rápidamente a interpretaciones alternativas o para buscar nuevas interpretaciones.

Una segunda hipótesis se refiere a los posibles efectos del bilingüismo en el procesamiento semántico. En tareas de asociación con niños monolingües sólo los niños mayores empiezan a dar muchas asociaciones que sitúan a la palabra estímulo en un sistema, esto es: asociaciones basadas en la similitud, el contraste, y la supraordenación. Todavía no sabemos cómo se desarrollan estas asociaciones categoriales. Si se desarrollan estrictamente a través de la inducción, a partir de la experiencia con palabras concretas en contextos variados, entonces no hay razones para pensar que los bilingües desarrollarán antes estas asociaciones categoriales. Es más, los bilingües tienen una experiencia relativamente limitada con las palabras concretas. Las palabras que oyen están divididas entre dos lenguas, de forma que tienen menos oportunidades de oír con frecuencia palabras concretas. Esta falta de 
experiencia con el vocabulario podría incluso retardar la aparición de asociaciones categoriales, si es que éste es el mecanismo por el cual empiezan a producirse.

Sin embargo, sí que podemos esperar una aparición precoz de asociaciones categoriales en los niños bilingües si la capacidad para asociar categorialmente palabras se intensifica con la experiencia frecuente de conflictos en la interpretación del significado de las palabras y de la estructura de frases.

Esta última predicción, es decir, el desarrollo precoz de asociaciones categoriales en los bilingües, hay que matizarla, ya que también es esperable que los bilingües tengan un déficit de vocabulario. Un conocimiento limitado de palabras podría dificultar la producción de asociaciones categoriales, a pesar de que el niño tenga una estrategia orientada en esta dirección.

Una tercera hipótesis es que la conciencia que tiene el niño bilingüe de la base sistemática de la estructura del lenguaje se generaliza a una capacidad mayor para analizar estructuras no verbales.

Hay evidencias empíricas de la influencia facilitadora del bilingüismo en la cognición. Peal y Lambert (1962) concluyeron que sus bilingües anglo-franceses de 10 años hacían mejor las pruebas de razonamiento intelectual que monolingües comparables. También encontraron que las capacidades de los bilingües estaban más diferenciadas. Sin embargo en este estudio no se controló la capacidad original, y había índices de que los bilingües del estudio podían ser más inteligentes al principio, ya que estaban más avanzados en la escuela que su grupo de comparación de la misma edad. Otro problema era que el criterio para considerar a un niño como bilingüe o monolingüe no implicaba una disyunción clara, y dependía también demasiado de puntuaciones de vocabulario. Si un efecto del bilingüismo es retardar el vocabulario, como se propone aquí, entonces el usar el vocabulario en una lengua menos conocida como criterio de bilingüismo supone un sesgo.

Anisfeld (nota 1) reanalizó los datos de Peal y Lambert para controlar la inteligencia, eliminando sujetos hasta que los grupos fueron comparables en el CI de Kuhlman-Anderson. Encontró que los bilingües seguían distinguiéndose por dar puntuaciones superiores en el Test de Matrices Progresivas de Raven, test considerado alto en «G». El procedimiento de Anisfeld no corrige el cuestionable criterio de selección del estudio original.

Los resultados de Ianco-Worrall (Ianco-Worrall, 1972; Worrall, nota 2), en su comparación de bilingües anglo-africanos de edades comprendidas entre 4 y 9 años con monolingües, fueron diferentes. Ella no encontró que sus bilingües fueran mejores en pruebas de capacidad para enfrentarse de una forma flexible con estructuras, o en pruebas de pensamiento operativo. Encontró, sin embargo, que sus bilingües estaban más orientados hacia estímulos significativos, y que eran más capaces de formarse un concepto de la palabra como símbolo arbitrario. El no haber encontrado diferencias lingüísticas en flexibilidad estructural podría 
estar reflejando su propio criterio de bilingüismo. No incluyó una prueba de capacidad para la traducción, aunque éste es el mejor índice de que el niño ha sido capaz de enfrentarse al conflicto interlingüístico. No describe con detalle su prueba sudafricana del CI, y por tanto no está clara su efectividad como control. Además, para la mayoría de sus bilingües cada uno de sus progenitores estaba asociado exclusiva y diferenciadamente con una de las dos lenguas. La predictibilidad completa del interlocutor permite disminuir el conflicto interlingüístico esencial, que se supone que constituye el factor motivacional básico en la situación cognitiva del niño bilingüe.

Para los bilingües usados en el estudio que vamos a describir más adelante, la lengua en que cualquier interlocutor concreto podría hablar al niño era menos predecible que en el estudio de Ianco-Worral. La inteligencia se controló por medio de tests normalizados. No se usó el nivel de vocabulario como criterio para determinar el bilingüismo. Las variables dependientes cognitivas se seleccionaron de acuerdo con las hipótesis descritas anteriormente.

\section{METODO}

\section{Sujetos}

Eran 96 niños divididos en cuatro grupos. Un grupo de niños anglo-hebreo-parlantes fue evaluado en los Estados Unidos, y un segundo grupo en Israel. Había dos grupos monolingües correspondientes, uno evaluado en los Estados Unidos y que hablaba sólo inglés, y el otro evaluado en Israel y que hablaba sólo hebreo. Las edades iban desde 5.4 a 8-6 años (media $=7.0$ años). La distribución por sexos era aproximadamente igual.

Todos los sujetos eran judíos de clase media. La ocupación de los padres eran académica o profesional. Según los padres, la edad media en la que comenzó el bilingüismo en la muestra de los Estados Unidos fue a los 2 años y medio, y a los 3 y medio en la muestra de los bilingües israelitas.

La mayoría de los bilingües de los Estados Unidos eran hijos de padres israelíes que vivían temporalmente en los Estados Unidos. La mayoría asistían a la escuela hebrea, en la cual se habla y enseña mediante el hebreo y el inglés. Oían también ambas lenguas en otros ambientes. La evaluación se realizó en tres escuelas hebreas del área de Chicago y una de Brooklyn, y también en los hogares de los niños. La evaluación de los monolingües de los Estados Unidos se realizó en una escuela pública suburbana de Chicago o en sus casas.

Los bilingües evaluados en Israel eran inmigrantes de Estados Unidos o Sudáfrica. Fueron evaluados en sus hogares, que estaban en los suburbios de Jerusalem y Tel Aviv. En estos barrios de inmigrantes son corrientes ambas lenguas, aunque las clases en la escuela son exclusivamente en hebreo. Los monolingües hebreos fueron evaluados en 
una escuela de Tel Aviv seleccionada, después de unas consultas con el ministerio de educación, a causa de su ubicación en un barrio de familias de origen europeo con un nivel ocupacional alto.

\section{Procedimiento}

Todos los niños fueron evaluados de forma individual y con tests en la lengua apropiada. A excepción de los bilingües israelíes, los cuales fueron evaluados por un psicólogo israelí, el investigador administró los tests. El orden de administración de los mismos era fijo.

La selección se realizó por medio de un test de traducción de 15 items, en el cual las frases a traducir eran alternadas de una lengua a la otra. El vocabulario era muy simple, pero la traducción de las frases exigía realizar algunos cambios en el orden de las palabras, en la construcción, y a veces ciertas adaptaciones idiomáticas. Si un sujeto monolingüe potencial mostraba cierta comprensión de alguna parte de las frases del segundo lenguaje, o si hablaba otra lengua extranjera, se eliminaba de la muestra. Los bilingües potenciales que no conseguían traducir más de dos frases a cuyas traducciones eran forzadas o literales se eliminaban también, aunque estos casos eran raros.

Dos puntuaciones determinaban el grado de bilingüismo de los sujetos seleccionados para su inclusión en la muestra de bilingües, una derivada de la prueba de traducción y la otra de un test de asociación de palabras bilingües de 18 items. Se encontró que estas dos eran las más representativas de cinco puntuaciones de bilingüismo procedentes de las pruebas con las que se hizo un análisis de regresión. Ambas puntuaciones seleccionadas se usaron para medir el equilibrio bilingüe, aunque también se incluyeron en la batería puntuaciones puras de los errores de traducción (errores acumulativos independientes de la dirección).

Para la puntuación del equilibrio bilingüe derivada del test de traducción, las frases de los niños se codificaban como «más» o «menos», en función de la lengua hacia la que se derivaba el error, y después se sumaban. Un equilibrio alto (es decir, poco sesgo) estaba representado por una puntuación cercana al cero. El acuerdo entre las puntuaciones independientes de un psicólogo israelí y el investigador fue $r=.96$.

En el test bilingüe de asociación de palabras, la mitad de las 18 palabras estímulo eran de cada una de las lenguas, en orden aleatorio. Se producía una puntuación alta en el equilibrio bilingüe cuando un sujeto respondía consistentemente en la misma lengua de la palabra estímulo concreta, o cuando las respuestas que cambiaban de lengua lo hacían aproximadamente igual en ambas direcciones. La correlación entre las dos medidas de capacidad de traducción usadas fue $r=.38$. Estas medidas se usaron como variables independientes en un análisis de varianza para determinar si la variación en el grado de 
bilingüismo dentro del grupo de bilingües seleccionados tenía un efecto independiente en el rendimiento, por encima del posible efecto principal del bilingüismo.

\section{Control de la inteligencia}

La puntuación en inteligencia se basó en una estimación hecha a partir de la Escala Wechsler de Inteligencia para Niños (Wechsler Intelligence Scale for Children; WISC). La puntuación total fue una ponderación de cuatro subtests del WISC: semejanzas, memoria de dígitos, figuras incompletas y ordenación de imágenes. La elección del WISC, así como de estos subtests concretos, respondió al compromiso entre dos criterios conflictivos: a) el estudio debía usar medidas bien normalizadas de lo que tradicionalmente se llama «inteligencia»; b) las pruebas usadas no debían estar influidas por el propio factor del bilingüismo.

La administración en Israel se realizó en hebreo para los monolingües y para aproximadamente la mitad de los bilingües israelíes, en función del juicio del evaluador, a partir del test de selección, de qué lengua hablaba mejor el niño. En los Estados Unidos se les administró a todos la forma inglesa, ya que la hebrea no estaba todavía preparada.

En el experimento principal se usó como control la puntuación estimada del WISC. En un análisis de varianza separado se encontró que las diferencias de grupo entre los grupos bilingüe y monolingüe en la puntuación total estimada del WISC, no era significativa cuando se promediaba sobre la nacionalidad. Este procedimiento se realizó sólo con la puntuación total, de forma que el desempeño en los subtests concretos no es necesariamente el mismo. El CI promedio era 113.

\section{Pruebas de flexibilidad en el uso de reglas sintácticas}

Test de transformaciones verbales. Warren y Warren (1966) encontraron que cuando un estímulo verbal se repite continuamente por medio de una cinta, los sujetos mayores de 6 años informan con frecuencia de cambios en lo que la voz parece decir. La ilusión se atribuyó a mecanismos de reorganización que se desarrollan para facilitar la percepción del habla continuada.

Los estímulos eran «flime» y «tress», ambas sin sentido para los niños evaluados. Los fonemas de estas palabras aparecen tanto en el inglés como en el hebreo, aunque el hablante usaba la pronunciación anglo-americana. Los estímulos se presentaban durante dos minutos a una velocidad de dos por segundo. Los cambios informados por el niño se registraban inmediatamente, y se cronometraban por medio de un reloj.

Se esperaba que los niños bilingües informasen de más transformaciones verbales. Los niños bilingües han tenido que aprender a 
interpretar el habla de dos tipos estructurales diferentes, y su interpretación inicial ha sido más incorrecta de lo que suele ser normal en el aprendizaje monolingüe. Es probable que esto haya conducido a una estrategia de reinterpretación rápida del patrón auditivo, que continúa hasta que se encuentra una interpretación satisfactoria. No hay una interpretación correcta posible en la presente tarea, ya que los estímulos no son significativos, con lo que las reorganizaciones continúan. Esta conducta es un desarrollo precoz, en el sentido de que es similar al responder adulto.

Test de substitución de simbolos. En esta tarea de siete items el niño tenía que sustituir una palabra significativa por otra, normalmente dentro de una estructura de frase fija. En los dos primeros items el niño tenía que admitir que una palabra podía sustituirse por otra, en vez de estar vinculada, de una forma inmanente o mágica, a su referente. Ejemplo: «Tú sabes que en inglés a esto se le llama aeroplano (el experimentador le muestra un avión de juguete). En este juego su nombre es turtle... ¿Puede volar el turtle? (respuesta correcta: sí). ¿Cómo vuela el turtle? (respuesta correcta: con sus alas)».

En los otros cinco items, la palabra de sustitución violaba obligatoriamente las reglas de selección del lenguaje. Ejemplo: «En este juego la forma de decir $Y O$, es decir macaroni. ¿Cómo decimos «yo tengo calor»? (respuesta correcta: «macaroni tengo calor»)».

Para responder correctamente el niño tiene que ser capaz de resistir la interferencia mutua entre el item sustituido y la estructura de la frase. Para superar esta resistencia hay que ignorar la función de referencia normal de la palabra sustituida, de forma que pueda ser tratada como una mera unidad dentro de un sistema de códigos. En caso contrario el niño dirá como mucho algo así como «macaroni tiene calor», en vez de «macaroni tengo calor».

El último item es el más complejo, pues en él hay que sustituir una parte fundamental del habla por otra menos importante. La palabra «clean» hay que sustituirla por «into», como en «the doll is going clean the bouse»"

Se esperaba que los bilingües fueran superiores en la sustitución de símbolos, debido a su forma, más analítica, de enfocar la estructura de las frases. Ya que las dos lenguas de los bilingües les proporcionan palabras para representar un objeto o evento dado, la idea mágica de que hay una relación determinada entre una palabra y su referente se disipa muy pronto. El éxito en la última parte de la prueba implica algo más, ya que para estos items hay que ignorar no sólo el significado semántico de palabras concretas sino también las reglas de selección que gobiernan las relaciones normales entre clases de palabras dentro de la frase, con objeto de tratarla como un código

(*) En esta frase, el cambiar clean por into cambia el sentido de is going: con clean se refiere a una acción que está a punto de comenzar (la muñeca va a limpiar la casa), y con into se refiere a una acción que se está realizando ya (la muñeca está entrando en la casa) (N. del T.). 
abstracto arbitrario. Habiendo experimentado más de un sistema de códigos de lenguaje, el bilingüe tendrá una libertad mayor para abandonar, cuando es necesario, las reglas de un sistema de lenguaje concreto por un conjunto diferente de reglas.

\section{Pruebas relativas al conocimiento semántico}

Test de asociaciones paradigmáticas. Las asociaciones paradigmáticas son aquellas que pertenecen a la misma parte del habla que la palabra estímulo, en oposición a las asociaciones sintagmáticas, las cuales podrían seguir a la palabra estímulo en una frase normal. Las respuestas paradigmáticas también tienden a categorizar a la palabra estímulo en alguna dimensión conceptual. Cuanto mayor sea la madurez dentro de las edades de nuestra muestra, antes empezarán a predominar las respuestas paradigmáticas sobre las respuestas sintagmáticas (Entwisle, Forsyth y Muus, 1964; Ervin, 1961). Los estímulos eran 18 palabras corrientes en las que estaban representadas todas las partes del habla. Se puso un énfasis mayor en los verbos y los adjetivos, ya que éstos no predisponen asociaciones de ninguno de los dos tipos de respuesta (Deese, 1965). La lista hebrea era una traducción de la inglesa, excepto los casos en los que la traducción era un homónimo.

Se les decía a los niños que diesen una sola palabra como respuesta a cada palabra estímulo. Al igual que en las otras tareas, ésta se les presentaba a cada sujeto en la lengua que cada bilingüe dominaba mejor, en función del juicio que se hacía a partir del test de traducción previo, y sólo se aceptaban respuestas en esta lengua.

Se esperaba que los bilingües diesen relativamente más asociaciones de tipo paradigmático, a causa de su desarrollo más maduro del sistema de clasificación semántica. Las palabras se asociarán más según categorías conceptuales que según las secuencias corrientes del habla. Por el contrario, la pobreza de vocabulario puede enmascarar este efecto, ya que las asociaciones paradigmáticas exigen el conocimiento de más palabras.

Peabody Picture Vocabulary Test. Se incluyó como medida del vocabulario más que con la finalidad con la que suele usarse, es decir, como test de inteligencia. Se aplicó sólo en inglés, ya que no existe una forma normalizada en hebreo, y sólo a las muestras de los Estados Unidos. Se esperaba que los bilingües diesen puntuaciones menores en el PPVT. Normalmente los bilingües tienen que aprender dos nombres diferentes para cada referente, uno de cada lengua. Por tanto, cualquier palabra concreta de una de las dos lenguas la habrá oído con menos frecuencia en su experiencia y estará peor aprendida. 


\section{Pruebas de comprensión de sistemas no verbales}

Transposición de matrices y designación de dimensiones. En la tarea original de Bruner y Kenney (1966) se presentaban al niño nueve cilindros que variaban según tres pesos y tres tamaños de diámetro, de tal forma que formaban una matriz de $3 \times 3$. Se le pedía al niño que transpusiese la matriz y la describiese. En el presente estudio se añadieron matrices intermedias con objeto de presentar el problema en pasos más pequeños. Se codificaron separadamente las respuestas descriptivas y las de transposición.

En los pasos 1 y 2 se presentaban al niño matrices de $2 \times 2$. En el paso 1 se le pedía al niño que describiese la forma en que los dos cilindros superiores eran «iguales» entre sí (pesaban igual), y en qué diferían entre sí (diferían en diámetro). Luego se les preguntaba en qué se parecían y en qué se diferenciaban los dos cilindros de la columna izquierda. A las respuestas se les adjudicaba de cero a cuatro puntos, según el número de descripciones correctas. Ya que cada cilindro aparecía en los dos conjuntos que el niño tenía que describir, éste tenía la oportunidad de darse cuenta de que cada objeto puede participar de dos dimensiones simultáneamente.

En el paso 2 se les presentaba una nueva matriz $2 \times 2$ en la que uno de los espacios estaba vacío. El niño tenía que elegir cuál de varios cilindros era el correcto para completar la matriz. La puntuación del niño en transposición aumentaba según que su elección fuese correcta en ninguna, una, o las dos dimensiones. Entonces el experimentador corregía las elecciones erróneas colocando el cilindro correcto en el espacio en cuestión mientras retiraba los otros, pero manteniéndolos a la vista. Entonces se les preguntaba a todos los sujetos por qué ese cilindro era el correcto. La puntuación en descripción se aumentaba según que el niño especificase ninguna, una, o ambas dimensiones.

En el paso 3 se les presentaba una matriz de $3 \times 2$ y se les pedía que la transpusieran. Si su ejecución era incorrecta, el experimentador la corregía cambiando los cilindros de alrededor. La puntuación se asignó de nuevo en base al número de dimensiones correctas. Entonces con todos los sujetos el experimentador apuntaba claramente a cada fila y columna de la matriz correctamente transpuesta, para indicarle al niño que en ella había un sistema regular. En este paso no había puntuación en descripción.

En la matriz final $3 \times 3$ la puntuación en descripción dependía de la capacidad para describir la diagonal de la matriz en la que las dimensiones variaban en la misma dirección. Se quitaba esta diagonal de la matriz y se le pedía al niño que dijese en qué eran diferentes entre sí los tres cilindros de la diagonal. En esta diagonal un cilindro era a la vez el más alto y el más ancho, el siguiente era el intermedio en ambas dimensiones, y el tercero era el más corto y el de menor diámetro. La puntuación en descripción variaba según que la progresión se describiese de una forma vaga y adimensional, que se confun- 
diesen las dos dimensiones, que sólo se describiese una dimensión aunque de forma consistente o que se describiesen ambas dimensiones de forma coherente y sin confundirlas. Las puntuaciones más altas correspondían a una estrategia más analítica, ya que el niño en este caso clasificaba las dimensiones como independientes en una situación que no requería una respuesta explícita de este tipo. Sólo si el niño ha analizado correctamente la matriz como un sistema y ha considerado las unidades de la diagonal con relación a la matriz mayor, dará el tipo superior de respuesta.

Cuando el niño usaba términos tales como «grande, medio, pequeño», se le preguntaba sobre ellos, para poder determinar si estaba refiriéndose a las dos dimensiones (y no sólo a la del peso), en cuyo caso se le daba una puntuación más alta en descripción. También podían indicarse las dimensiones señalando, en vez de con palabras, cuando estaba claro que la referencia era a una dimensión concreta.

Por último, se le pedía al niño que hiciese una transposición de la matriz $3 \times 3$. En el análisis final había dos puntuaciones diferentes de la transposición: una para la transposición $3 \times 3$ sola, y otra que incluía tanto ésta como la transposición de la matriz $3 \times 2$ y la capacidad para colocar el cilindro correcto en la posición vacía de la matriz $2 \times 2$.

Como ya he dicho antes, la hipótesis era que los niños bilingües desarrollan un nivel inusual de comprensión de sistemas a nivel de sintaxis, como resultado de su experiencia especial con el lenguaje. La predicción era que esta comprensión se generaliza y aparece en forma de un alto rendimiento en transposición de matrices, lo cual requiere una capacidad especial para analizar sistemas no verbales.

Test de Matrices Progresivas de Raven (partes $\mathrm{AB}$ y $\mathrm{B}$ ). Las destrezas que se necesitan en esta tarea son similares a las que se necesitan para la transposición de matrices. Ambas implican lo que Piaget llama «clasificación multiplicativa», en la cual hay que atender simultáneamente a dos dimensiones de un sistema. En las Matrices Progresivas de Raven la predicción era también que los bilingües realizarían mejor la tarea.

El problema del uso de estas pruebas, especialmente las Matrices Progresivas de Raven, es que es probable que su correlación con medidas de inteligencia general sea alta. Ya que la inteligencia general, según la medida del WISC, se controlaba estadísticamente en este estudio, la probabilidad de que apareciesen diferencias entre grupos en estos tests era pequeña. Aun así, la inteligencia general había que controlarla de alguna manera, ya que de lo contrario un resultado que indicase un rendimiento superior de los bilingües no sería significativo. Se decidió incluir de todas formas la transposición de matrices y el Test de Matrices Progresivas de Raven, con la posibilidad de que apareciesen diferencias. Mientras que no hay seguridad de que la combinación de los subtests del WISC que componen la medida de inteligencia en este estudio sea independiente de la comprensión de 
sistemas, al menos la combinación exige una variedad de destrezas intelectuales, en contraste con las tareas de matrices, las cuales se centran casi exclusivamente en el análisis de sistemas.

Procedimiento estadístico. El programa usado fue un análisis univariado y multivariado de varianza y covarianza, programa en FORTRAN IV, versión 4 (Finn, nota 3). El análisis principal tenía cuatro variables independientes: edad (de $5-4$ a 6-11 versus de 7-0 a 9-6); sexo; lenguaje (bilingüe versus monolingüe); y localización (Israel versus Estados Unidos). Las puntuaciones del CI en el WISC se controlaron tratándolas como covariadas, controlando independientemente cada subtest y la puntuación total. Otro análisis abarcaba sólo a la muestra de los Estados Unidos, ya que el PPVT se administró sólo en los Estados Unidos. El análisis final comprobaba si el grado de bilingüismo y los factores de edad y edad de partida estaban relacionados con las diversas variables dependientes medidas.

\section{RESULTADOS}

\section{Resultados referentes al uso de reglas sintácticas}

1. Los bilingües hicieron significativamente más transformaciones verbales, informaron de más formas diferentes, y comenzaron a oírlas antes, tal y como muestra la Tabla 1 . No hubo diferencias entre los grupos en el número de formas significativas oídas en ambas lenguas.

Tabla 1. Medidas del Test de Transformaciones Verbales.

\begin{tabular}{|c|c|c|c|c|}
\hline & $\begin{array}{c}\text { Media } \\
\text { Bilingüe }\end{array}$ & $\begin{array}{l}\text { Media } \\
\text { Monolingüe }\end{array}$ & DT & F \\
\hline $\begin{array}{l}\mathrm{N} .^{\circ} \text { total de formas oídas } \ldots \ldots \\
N .^{\circ} \text { de formas diferentes } \ldots \\
\mathrm{N}^{\circ} \text { de formas en los primeros }\end{array}$ & $\begin{array}{r}10.79 \\
6.97\end{array}$ & $\begin{array}{l}7.69 \\
4.88\end{array}$ & $\begin{array}{l}4.09 \\
2.42\end{array}$ & $\begin{array}{l}9.86 * \\
14.76 * *\end{array}$ \\
\hline 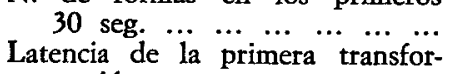 & 3.00 & 2.12 & 1.09 & $12.63 * *$ \\
\hline $\begin{array}{llllllll}\text { mación } & \ldots & \ldots & \ldots & \ldots & \ldots & \ldots\end{array}$ & 14.01 & 22.96 & 11.30 & $12.58 * *$ \\
\hline
\end{tabular}

Nota: Grados de libertad $=1.75 ;{ }^{*} \mathrm{p}<.01 ;{ }^{\star \star} \mathrm{p}<.001$.

A medida que aumentaba la edad se producía mayor actividad transformadora, según indica el número total de transformaciones oídas, $\mathrm{F}(1,75)=12.65, \mathrm{p}<.001$. Todas las otras medidas estaban también significativamente relacionadas con la edad, excepto la latencia de la primera transformación. Esto significa que, al dar más transformaciones, los bilingües reaccionan más como los niños mayores y los jóvenes adultos, lo cual nos indica que tienen un desarrollo precoz. La única diferencia es que en las edades superiores los mo- 
nolingües oyeron más palabras significativas, $\mathrm{F}(1,75)=9.34, \mathrm{p}<0.1$, mientras que los bilingües no.

En los bilingües una actividad transformadora alta se relacionaba con CIs altos, mientras que esto fue significativamente menos cierto en los monolingües, tal y como se manifiesta en el efecto significativo de la interacción entre el CI y las condiciones de lenguaje en el número total de transformaciones verbales, $F(1,48)=5.40, p<.05$. De esto se desprende que una orientación temprana hacia la reorganización es especialmente adaptativo para los bilingües.

2. En la tarea de sustitución de símbolos los bilingües fueron significativamente superiores, $F(1,84)=7.72, \mathrm{p}<.01$. El análisis de items mostró que los bilingües eran superiores tanto en la primera parte, que dependía de la liberación de las palabras como eventos «mágicos», como en los items posteriores, que requerían una cierta capacidad para dejar a un lado, cuando es necesario, las reglas de selección y categorización de la sintaxis de las frases.

\section{Resultados referentes a la comprensión semántica}

3. En las asociaciones paradigmáticas los resultados no fueron exactamente los esperados. Los bilingües no dieron más asociaciones paradigmáticas que los monolingües. Como puede verse en la Tabla 2,

Tabla 2. Puntuaciones en la prueba de asociación de palabras.

\begin{tabular}{|c|c|c|c|}
\hline & $\begin{array}{c}\text { Media } \\
\text { Bilingüe }\end{array}$ & $\begin{array}{l}\text { Media } \\
\text { Monolingüe }\end{array}$ & $\mathrm{F}$ \\
\hline \multicolumn{4}{|l|}{ Tipo de respuesta $\left({ }^{(}\right)$} \\
\hline $\begin{array}{llllllll}\text { Paradigmática } & \ldots & \ldots & \ldots & \ldots & \ldots & \ldots & \ldots \\
\text { Sintagmática }\left({ }^{\circ}\right) & \ldots & \ldots & \ldots & \ldots & \ldots & \ldots\end{array}$ & $\begin{array}{l}7.80 \\
1.90\end{array}$ & $\begin{array}{l}7.59 \\
0.37\end{array}$ & $\begin{array}{c}0.74 \\
10.86\end{array}$ \\
\hline \multicolumn{4}{|l|}{ Latencia } \\
\hline $\begin{array}{llllllll}\text { Paradigmática } & \ldots & \ldots & \ldots & \ldots & \ldots & \ldots & \ldots \\
\text { Sintagmática } & \ldots & \ldots & \ldots & \ldots & \ldots & \ldots & \ldots\end{array}$ & $\begin{array}{l}5.35 \\
6.32\end{array}$ & $\begin{array}{l}4.12 \\
8.51\end{array}$ & $\begin{array}{l}5.25 * \\
0.77\end{array}$ \\
\hline
\end{tabular}

Nota: grados de libertad $=1.75$;

() N. de respuestas máxima $=18$.

(b) El n. de respuestas sintagmáticas no se incluyó en el programa de computador, pero una prueba $t$ aparte de las diferencias entre las medias no fue significativa, $\mathrm{t}(91)=0.10, \mathrm{p}>.05$

$\star \mathrm{p}<0.05$

$\star * \mathrm{p}<.01$

no se encontraron diferencias de grupo ni en las respuestas sintagmáticas ni en las paradigmáticas. Los bilingües dieron significativamente más respuestas «de ruido», un tipo de respuesta inmadura que indicaba que la tarea de asociación era especialmente difícil para ellos. Otro índice de esta dificultad fue que cuando los bilingües daban respuestas paradigmáticas su latencia era mayor, indicando un esfuerzo más grande de búsqueda que el de los monolingües. 
4. Los bilingües fueron significativamente peores en el PPVT, $t(54)=3.20, p<.001$. La Tábla 3 muestra que en los monolingües la puntuación del CI en el WISC es muy cercana a la puntuación en vocabulario, mientras que en los bilingües hay una gran discrepancia. Sus puntuaciones en vocabulario son considerablemente menores que sus puntuaciones en el WISC.

Tabla 3. CI medio en vocabulario comparado con el CI medio en el WISC (estimado).

\begin{tabular}{|c|c|c|}
\hline & $\begin{array}{l}\text { Media } \\
\text { Bilingüe }\end{array}$ & $\begin{array}{c}\text { Media } \\
\text { Monolingüe }\end{array}$ \\
\hline Vocabulario en Peabody $\ldots \ldots \ldots$ & 100.50 & 111.38 \\
\hline CI en el WISC $\ldots \begin{array}{lllll} & \ldots & \ldots & \ldots & \ldots\end{array}$ & 118.45 & 115.50 \\
\hline
\end{tabular}

Nota: Estos datos se refieren sólo a los grupos de los Estados Unidos, ya que sólo a éstos se les administró el Peabody.

La relación entre las puntuaciones en el PPVT (Prueba de Peabody) y en la prueba de asociaciones paradigmáticas ayuda a clarificar por qué los bilingües no respondieron como se esperaba en el test de asociaciones. Se realizó un análisis de varianza aparte con la muestra de los Estados Unidos en el que el Peabody, el bilingüismo-monolingüismo, y el CI del WISC eran variables independientes, y el Test de Matrices Progresivas se trataba como un test de inteligencia y se controlaba como covariado. Las variables dependientes eran la sustitución de símbolos y varias medidas de las pruebas de transformaciones verbales y de asociaciones paradigmáticas. En estas condiciones sólo las medidas de asociación paradigmática se vieron afectadas por el Peabody como variable independiente. Cuando la condición lingüística y el Peabody se combinaban como variables independientes, el efecto sobre el número de asociaciones paradigmáticas era casi significativo, $\mathrm{F}(1,48)=2.03, \mathrm{p}=16(\mathrm{~F}=3.55, \mathrm{p}=.06$ «step downis), y el efecto sobre la latencia de las asociaciones paradigmáticas era significativo, dependiendo de qué medida se usaba, $\mathrm{F}(1,48)=3.62, \mathrm{p}=.06,(\mathrm{~F}=$ $=4,41, \mathrm{p}<.05$ «step down»). Según las medias, las puntuaciones bajas en el PPVT estaban asociadas con un número pequeño de respuestas paradigmáticas y una latencia alta de las mismas. Los bilingües dieron puntuaciones significativamente menores en vocabulario. Esta puede ser la causa de no haber encontrado el número alto de tespuestas paradigmáticas que se esperaba.

\section{Resultados referentes a la comprensión de sistemas no verbales}

5. Los bilingües fueron significativamente mejores en la parte descriptiva de la tarea de transposición de la matriz. Esto es, eran 
más capaces de aislar y especificar las dimensiones subyacentes en la matriz, $\mathrm{F}(1,75)=8.74, \mathrm{p}<.01$, media de los bilingües $=4.09$, media de los monolingües $=3.44$. Independientemente de que respondieran con palabras o con gestos, los bilingües eran capaces de aislar mejor las dimensiones de la matriz. Con respecto a esto, la orientación que se encontró en los bilingües hacia el análisis con material estrictamente verbal, se generaliza al no verbal.

6. En los resultados de la tarea de las Matrices Progresivas de Raven y en la parte de ejecución de la tarea de transposición de matrices, que es similar a la tarea de las Matrices Progresivas de Raven, no se encontraron diferencias de grupo.

7. Se analizaron los errores en la tarea de las matrices de Raven. No se encontraron diferencias ni en los errores parciales (en los cuales sólo se tomaba en consideración una de las dos dimensiones relevantes), ni en los de orientación espacial, ni en los de irrelevancia, pero sí se encontraron diferencias significativas de grupo en los errores que se basaban en fallos de examen, $\mathrm{F}(1,84)=4.10, \mathrm{p}<.05$. Cuando cometen este tipo de errores los niños simplemente eligen los items adyacentes al que falta, en vez de examinar todos los items posibles. Evidentemente, los bilingües se enfrentaron a la tarea con una estrategia de solución de problemas más definida o, al menos, con una mayor atención a las posibles diferencias y a su naturaleza, lo cual fue también evidente en algunas de las otras tareas.

\section{Resultados referentes al grado de bilingüismo}

8. El grado de bilingüismo no se relacionaba con las puntuaciones cognitivas. Esto es, por encima del estricto criterio de desempeño en la traducción, que se usó para seleccionar a los niños que iban a constituir la muestra bilingüe, no resultó importante el grado de perfección y de exactitud de la traducción.

\section{DISCUSION}

Los resultados de la prueba de transformaciones verbales, y en cierto sentido los de la prueba de sustitución de símbolos, apoyan la hipótesis de que los bilingües están más preparados para reorganizar sus percepciones. El desempeño en esta última prueba puede tomarse también como un índice de la comprensión de la arbitrariedad de la estructura sintáctica por parte de los bilingües. Los bilingües fueron más capaces de tratar a las palabras como unidades «des-semantizadas» dentro de un sistema de codificación mayor, y de cambiar las reglas del sistema, tal y como exigía la prueba.

Podría argumentarse que los bilingües eran capaces de ignorar las reglas sintácticas normales, y por tanto de realizar bien la sustitución de símbolos, sólo porque no dominan esas reglas lo suficiente como 
para que les estorben. Este argumento no es sostenible, ya que se administró a esta muestra un test tipo Berko (1958) de la capacidad para porporcionar adiciones morfémicas apropiadas a palabras sin sentido, sin encontrarse diferencias entre los grupos. Los paradigmas evaluados fueron: la pluralización, los tiempos pasados, el comparativo, el superlativo, la pasiva, el cambiar un adjetivo por un nombre abstracto, y el cambiar un adjetivo por un adverbio. Este resultado significa que los bilingües tienen un control de los paradigmas gramaticales ordinarios tan bueno como el de los monolingües. La implicación de los resultados en la sustitución de símbolos es, por consiguiente, que los bilingües se enfrentan a la sintaxis de una forma muy analítica y van más allá del dominio de las reglas sintácticas ordinarias.

En cuanto al nivel semántico, la predicción era que en una prueba de asociación los bilingües darían más asociaciones conceptuales que dependientes de la probabilidad secuencial. Esto se basaba en la idea de que la unión de una palabra a una frase concreta en una lengua concreta produce una interferencia de la estructura de la frase en la otra lengua, lo cual lleva a los bilingües a depender de aspectos de las palabras que son más constantes, es decir, sus significados conceptuales, tal y como vienen representados en las asociaciones paradigmáticas.

El fracaso de esta predicción se debió, al menos en parte, al pobre vocabulario de los bilingües, que hizo que para ellos la tarea de asociación fuese más difícil. Las respuestas paradigmáticas en particular fueron especialmente difíciles para ellos, ya que la latencia de éstas fue significativamente más alta en los bilingües que en el otro grupo, mientras que en los otros tipos de respuesta no hubo diferencias en la latencia (tabla 2). Es interesante el que a pesar de esta dificultad especial con las respuestas paradigmáticas, los bilingües diesen no obstante tantas respuestas de éstas como los monolingües. Quizás esto indica que los bilingües estaban haciendo un esfuerzo especial para producir este tipo de asociaciones, tal y como se supuso.

Después de ver la pobreza de vocabulario de los bilingües, podría haber sido más válido usar un tipo de prueba de asociación en la que se requiriesen juicios, más que producción de palabras. Ianco-Worral (1972) encontró que sus niños bilingües empezaban a seleccionar a una edad más temprana que los niños monolingües la palabra asociada semánticamente cuando se les pedía que determinasen cuál de entre dos palabras, una asociada semánticamente y otra fonéticamente, se parecía más a la palabra estímulo. En este estudio el número significativamente mayor de asociaciones fonéticas de los bilingües fue probablemente el resultado de los problemas de producción. Hay razones para pensar que esto puede disminuir con la edad y la oportunidad de tener más experiencia con la palabra.

Con respecto a la hipótesis de que la atención a la estructura y la facilidad para la reorganización que manifiestan los bilingües en relación con la estructura del lenguaje aparecerá también en sus percepciones 
de otros tipos de estructuras, los datos la apoyan en un aspecto pero no en otro. Las dos tareas que se relacionaban con esta hipótesis eran la transposición de matrices y las Matrices Progresivas de Raven. En ninguna de las dos fueron superiores los bilingües en la reorganización de las matrices. Sin embargo, en la parte verbal de la prueba de transposición de matrices, en la cual el niño tiene que aislar las dimensiones básicas que subyacen en la matriz los bilingües fueron significativamente superiores. La presencia de una estrategia analítica viene indicada también por el hecho de que los bilingües tendían a examinar más que los monolingües el campo de las respuestas posibles en las Matrices de Raven.

Parece, por tanto, que hay una cierta generalización de la estrategia de atender a la estructura del lenguaje, provocando una orientación más analítica hacia las estructuras, pero que ésta no implica una capacidad general para la reorganización de estructuras. En estructuras no verbales parece tomar la forma de una atención hacia las dimensiones básicas implicadas y al abanico de formas implicadas. Este aspecto es esencial pero no suficiente, por sí mismo, para la organización de un sistema. Este tipo de estrategia atencional sería más útil en situaciones en las que lo importante es darse cuenta de los detalles principales.

Será necesario realizar nuevos estudios para determinar si el efecto del bilingüismo en la cognición que hemos encontrado en este estudio depende de un tipo especial de situación de aprendizaje bilingüe, o de una actitud especial del entorno cultural del niño.

Los resultados de este estudio pueden resumirse así: hay dos estrategias que caracterizan las pautas de pensamiento de los bilingües con relación al material verbal: la tendencia a detectar la estructura, y la tendencia a la reorganización. Los bilingües buscan las dimensiones que subyacen a los patrones a los que se enfrentan. Las pautas que buscan son fundamentalmente lingüísticas, pero este proceso opera también con pautas visuales, como en su aptitud para aislar las dimensiones de una matriz. Sin embargo, con material visual no apareció la destreza especial para la reorganización.

Los bilingües de este estudio se han enfrentado a una edad muy temprana con un entorno verbal de complejidad anormal, en el que el orden subyacente es difícil de descubrir, ya que las reglas pertenecen a dos estructuras en vez de a una. Como resultado, parecen haber desarrollado una facilidad especial para buscar las reglas y para determinar cuáles son necesarias en cada circunstancia. Los datos apoyan la conclusión de que esta facilidad constituye un rasgo cognitivo que aparece más claramente que en la situación audio-verbal original, aunque atenuada. 


\section{Notas de referencia}

1. ANISFELD, E., A comparison of the cognitive functioning of monolinguals and bilinguals, Tesis doctoral no publicada, McGill University, 1964.

2. Worrall, A., Bilingualism and cognitive development. Tesis doctoral no publicada, Cornell University, 1970.

3. FINN, J. D., Multivariance-univariate and multivariate analysis of variance and covariance: a ForTArN IV program, 4. No publicado. State University of New York at Buffalo, Department of Educational Psychology, 1968.

\section{Referencias}

Berko, J., «The child's learning of English morphology», Word, 1958, 14, pp. 150-177.

BLomm, L., Language development: form and function in emerging grammars, Cambridge, Mass., M.I.T. Press, 1970.

Bruner, J., y Kenney, H., On multiple ordering. En J. Bruner, R. Olver, P. Greenfield, et al., Studies in cognitive growth, Nueva York, Wiley, 1966.

DEESE, J., The structure of associations in language and thought, Baltimore, John Hopkins Press, 1965.

ENTwisle, D.; Forsyth, D., y MuUs, R., «The syntactic-paradigmatic shift in children's word associations», Journal of Verbal Learning and Verbal Bebavior, 1964, 3, pp. $19-29$.

ERvin, S., "Changes with age in the verbal determinants of word-associations», American Journal of Psychology, 1961, 74, pp. 361-372.

ERvin-Tripp, S., «Some strategies for the first two years》. En T. Moore (Ed.), Cognitive development and the acquisition of language, Nueva York, Academic Press, 1973.

InNCo-Worralt, A., «Bilingualism and cognitive development», Cbild Development, 1972, 43, pp. $1390-1400$.

Peal, E., y LAMBerT, W., «The relation of bilingualism to intelligence», Psychological Monographs, 1962, 76 (27, Whole No. 546).

WARREN, R. M., y WARREN, R. P., «A comparison of speech perception in childhood, maturity, and old age by means of the verbal transformation effetc», Journal of Verbal Learning and Verbal Bebavior, 1966, 5, pp. 142-146.

WeInRerch, U., Languages in contact, Nueva York, Linguistic Circle of New York, 1953. 